\title{
Properly embedded minimal annuli bounded by a convex curve
}

\author{
Joaquín Pérez* Antonio Ros*
}

May 30, 2000

\begin{abstract}
We prove that given a convex Jordan curve $\Gamma \subset\left\{x_{3}=0\right\}$, the space of properly embedded minimal annuli in the halfspace $\left\{x_{3} \geq 0\right\}$, with boundary $\Gamma$ is diffeomorphic to the interval $[0, \infty)$. Moreover, for a fixed positive number $a$, the exterior Plateau problem that consists of finding a properly embedded minimal annulus in the upper halfspace, with finite total curvature, boundary $\Gamma$ and a catenoid type end with logarithmic growth $a$ has exactly zero, one or two solutions, each one with a different stability character for the Jacobi operator.
\end{abstract}

Mathematics Subject Classification: Primary 53A10, Secondary 49Q05, 53C42.

Key words and phrases: Minimal surface, logarithmic growth, Jacobi operator.

\section{Introduction.}

This paper is devoted to understand the space of properly embedded minimal annuli in the upper halfspace of three-dimensional Euclidean space, with boundary given by a (fixed) convex Jordan curve $\Gamma$ in the boundary of this halfspace. Under these conditions, Collin's result [2] implies that such annuli have finite total curvature, and so, they are asymptotic to a horizontal plane or to a half-catenoid. By the maximum principle, in the first case the surface coincides with the exterior of $\Gamma$ in the plane $\left\{x_{3}=0\right\}$. In the second case, the logarithmic growth of the asymptotic half-catenoid is the main parameter associated to the surface.

Recall that a noncompact minimal surface $M$ is called stable if for each compact subdomain of $M$ and respect to any nontrivial normal variation fixing its boundary, the second derivative of the area functional is nonnegative. Otherwise, $M$ is called unstable. When $M$ is complete, has finite total curvature and compact boundary, its Jacobi operator $L=\Delta+\|\nabla N\|^{2}, N$ being the Gauss map of $M$, extends to the compactification $\bar{M}$. In this setting, stability translates into that the first (Dirichlet) Jacobi eigenvalue is nonnegative.

\footnotetext{
${ }^{*}$ Research partially supported by a DGYCYT Grant No. PB94-0796.
} 
Finally, $M$ is called strictly stable if the its first Jacobi eigenvalue is positive. Our main Theorem describes this space of minimal annuli in terms of the logarithmic growth and the stability character:

Theorem. Let $\Gamma \subset\left\{x_{3}=0\right\}$ be a smooth convex Jordan curve. Then, the space $\mathcal{M}$ of properly embedded minimal annuli in the upper halfspace with boundary $\Gamma$, is a connected one-dimensional real analytic manifold diffeomorphic to $[0, \infty)$. If we start from the flat surface $M_{0} \in \mathcal{M}$ given by the exterior of $\Gamma$ in $\left\{x_{3}=0\right\}$ (which corresponds to zero in the representation of $\mathcal{M}$ as $[0, \infty))$, then we obtain the stable surfaces in $\mathcal{M}$ by increasing the logarithmic growth, until reaching a unique maximum which corresponds to a surface with first Jacobi eigenvalue zero. Besides this critical value, the surfaces in $\mathcal{M}$ become unstable and are parametrized by decreasing the logarithmic growth until the value zero, where elements in $\mathcal{M}$ tend to the plane $\left\{x_{3}=0\right\}$ covered twice on the interior of $\Gamma$ and once on the exterior of $\Gamma$ in such plane.

The unique maximum $a_{0}$ of the logarithmic growth in $\mathcal{M}$ is also the extremal value for the "exterior Plateau problem": for $a>a_{0}$ there are no properly immersed minimal surfaces in the upper halfspace with boundary $\Gamma$ and one embedded end of finite total curvature with logarithmic growth $a$ (with or without branch points); for $a=a_{0}$, the only solution to this Plateau problem is the critical annulus in $\mathcal{M}$, while for $0<a<a_{0}$ there exist exactly two annular solutions to the Plateau problem with this boundary and logarithmic growth, one strictly stable and one unstable. In this last case, perhaps $\Gamma$ is the boundary of other solutions of the Plateau problem with higher topology. For further references about the exterior Plateau problem, see Krust [6] and Tomi and Ye [20].

In [9], Meeks and White obtained similar results for the case of compact annuli bounded by two convex Jordan curves in parallel planes, see also [10] for related results. The proof of our results combines the ideas of Meeks and White with the approach developed by the authors in former works $[12,14,15]$ about smoothness of spaces of noncompact properly embedded minimal surfaces with both fixed topology and finite total curvature.

\section{Posing the problem.}

Let $\Gamma$ be a smooth convex Jordan curve in the plane $\left\{x_{3}=0\right\}$. We will call $\mathcal{M}$ to the space of all minimal annuli which are properly immersed in the upper halfspace $\left\{x_{3} \geq 0\right\}$, with finite total curvature, boundary $\Gamma$ and one embedded end. As we will see immediately, all surfaces in $\mathcal{M}$ are embedded. We also note that there are no properly embedded minimal surfaces in the upper halfspace, with finite total curvature, genus zero and more than one end. This follows from the vertical flux arguments by the authors in [13].

Each surface $M \in \mathcal{M}$ can be conformally parametrized by the punctured disk $\{0<$ $|z| \leq 1\}$ so that the end corresponds to $z=0$, Osserman [11]. We will call $\bar{M}$ to the 
compactified surface obtained by attaching the puncture to $M$. The finite total curvature assumption implies that the Gauss map of $M$ extends meromorphically to $\bar{M}$, taking a vertical value at the end, which we will suppose $e_{3}=(0,0,1)$ for the remainder of the paper. Moreover, the end must be asymptotic to a horizontal plane or to a vertical catenoid. It will be convenient for our purposes to have another conformal parametrization of $M$, by its Gauss map. We will call $M_{0}$ to the exterior of the curve $\Gamma$ in the plane $\left\{x_{3}=0\right\}$. The following Lemma is similar to some results contained in [9].

Lemma 1 For any nonflat surface $M$ in $\mathcal{M}$, we have

i) The end of $M$ is horizontal and of catenoid type.

ii) $M$ has no points with vertical normal vector.

iii) The level curve $\Gamma_{c}=M \cap\left\{x_{3}=c\right\}$ is strictly convex for each $c>0$. In particular, $M$ is embedded.

iv) The extended Gauss map is a conformal diffeomorphism between $\operatorname{Int}(\bar{M})$ and a domain of the sphere $\mathbb{S}^{2}$ containing exactly one vertical direction.

v) If the angle between $M$ and $M_{0}$ along $\Gamma$ is always smaller than $\pi / 2$, then $M$ is a global graph over $M_{0}$.

Proof. As $M$ is contained in the upper halfspace, its end must be horizontal. Denote by $x_{3}: M \rightarrow \mathbb{R}^{3}$ its third coordinate function. If the end of $M$ were planar, then $x_{3}$ would be a bounded harmonic function on a punctured disk with zero boundary values, hence constantly zero by the maximum principle. This proves $i$ ). On the other hand, $x_{3}$ is harmonic on $M$, has zero boundary values and a logarithmic singularity at the end, so, in terms of the $z$ coordinate, it is given by $x_{3}(z)=\alpha \log |z|$, for a negative real number $\alpha$. As this function has no critical points, we conclude ii). As the end is asymptotic to a vertical catenoid, $\Gamma_{c}$ is a strictly convex curve for $c$ greater than or equal to a certain $c_{0}>0$. The portion $M \cap\left\{0 \leq x_{3} \leq c_{0}\right\}$ is a minimal annulus bounded by two convex curves in parallel planes, hence all intermediate horizontal sections are strictly convex by a Theorem of Shiffman [19], and iii) is proved.

Next we will show that $N$ is unbranched at the interior of $\bar{M}$. For, fix $c>0$ and call $n, \kappa_{n}, \kappa$ to the unit normal vector to the planar curve $\Gamma_{c}$, its normal curvature and its planar curvature, respectively. As $\kappa_{n}=\kappa\langle n, N\rangle, \Gamma_{c}$ is strictly convex and $N$ is never vertical on $M$, we conclude that $\kappa_{n}$ does not vanish and hence, $N$ is a local diffeomorphism at the interior of $M$. As the end is of catenoid type, $N$ is also unbranched at the end.

To show iv), fix $c>0$. As the curve $\Gamma_{c}$ is strictly convex, it follows that the Gauss map $N$ applies $\Gamma_{c}$ diffeomorphically onto a (regular) Jordan curve separating the North and South poles of the sphere, which is starshaped respect to these points (note that the 
above property does not hold for the boundary curve $\Gamma$, which is convex but not necessarily strictly convex). Let $\bar{M}_{c}=\bar{M} \cap\left\{x_{3}>c\right\}$. The extended Gauss map is an open map on $\bar{M}_{c}$ that misses the value $(0,0,-1)$, and therefore $N$ applies $\bar{M}_{c}$ onto the component of $\mathbb{S}^{2}-N\left(\Gamma_{c}\right)$ that contains to $(0,0,1)$. Thus $N$ is a proper local diffeomorphism between $\bar{M}_{c}$ and $N\left(\bar{M}_{c}\right)$, which implies that $N$ is injective on $\bar{M}_{c}$. Taking $c$ arbitrarily small, we conclude $i v)$.

Concerning $v$ ), as $M$ meets $M_{0}$ with angle smaller than $\pi / 2$ and the value of the Gauss map $N$ at the end is $(0,0,1)$, it follows that $N(\Gamma)$ is a curve contained in the open upper hemisphere. By $i v), N(M)$ is also contained in the upper hemisphere, thus the orthogonal projection $\pi$ of $M$ on the plane $\left\{x_{3}=0\right\}$ is a local diffeomorphism. After gluing smoothly $M$ with a suitable disk along $\Gamma, \pi$ becomes a proper local diffeomorphism onto the whole plane $\left\{x_{3}=0\right\}$, hence a global diffeomorphism. In particular, $M$ must be a graph over $M_{0}$.

For a finite union of disjoint smooth Jordan curves $C \subset \mathbb{R}^{3}$, consider the space $\mathcal{M}(k, n, C)$ of properly embedded minimal surfaces with finite total curvature, genus $k$, $n$ horizontal ends and boundary $C$. Given $M \in \mathcal{M}(k, n, C)$, the linear space $\mathcal{J}(M)$ of infinitesimal deformations of $M$ in $\mathcal{M}(k, n, C)$ is the set of Jacobi functions on $M$ that have a logarithmic singularity at the ends and vanish at the boundary. The authors studied in [15] the smoothness of this space, showing that if the set of Jacobi functions on $M$ vanishing at the boundary and at the ends reduces to zero (a surface with this property will be called, as usual, nondegenerate), then the space $\mathcal{M}(k, n, C)$ is a real analytic $n$ dimensional manifold in a neighborhood of $M$. They also proved that any stable surface in $\mathcal{M}(k, n, C)$ is nondegenerate. In the particular case $k=0, n=1$ and $C=\Gamma$ for a convex Jordan curve $\Gamma$ as in our setting, a surface $M \in \mathcal{M}(0,1, \Gamma)$ is either the exterior of $\Gamma$ in the plane $\left\{x_{3}=0\right\}$ (denoted as $M_{0}$ ) or its logarithmic growth is positive (resp. negative), in which case the maximum principle implies that $M$ is contained in the upper (resp. lower) halfspace. So, the space $\mathcal{M}(0,1, \Gamma)$ consists of the surfaces in $\mathcal{M}$ and their reflected images respect to the plane $\left\{x_{3}=0\right\}$.

Next we will show that nondegeneracy is shared by all surfaces $M \in \mathcal{M}$. Suppose that $M$ is not flat, and note that the parametrization of $M$ by its Gauss map transforms the Jacobi equation $\Delta u+\|\nabla N\|^{2} u=0$ on $M$ in the equation $\Delta u+2 u=0$ on its spherical image, which lets us identify $\mathcal{J}(M)$ with

$$
\mathcal{J}(M)=\left\{\begin{array}{l|l}
u: \Omega-\left\{e_{3}\right\} \rightarrow \mathbb{R} & \begin{array}{c}
\Delta u+2 u=0 \quad \text { in } \Omega-\left\{e_{3}\right\} \\
u=0 \quad \text { in } \partial \Omega \\
u \text { has a logarithmic singularity at } e_{3}
\end{array}
\end{array}\right\},
$$

where $\Omega$ is the image set of the extended Gauss map. The above description of $\mathcal{J}(M)$ is clear provided that $\Gamma$ is strictly convex, in which case $\Omega$ has smooth boundary and $N$ is a 
diffeomorphism between $\bar{M}$ and the closure of $\Omega$. When $\Gamma$ is merely convex, this representation for $\mathcal{J}(M)$ remains valid, and the proof follows from the fact that the Sobolev norm on $\Omega$, translated to $\bar{M}$ through the Gauss map, is given by $v \mapsto \int_{\bar{M}}\left(\|\nabla N\|^{2} v^{2}+\|\nabla v\|^{2}\right) d A$, which is equivalent in the subspace of all compactly supported smooth functions $u$ on $\bar{M}$ to the usual Sobolev norm. Thus, stability and nondegeneracy questions can be answered only by reasoning with the Laplace-Beltrami operator of the standard metric on proper domains of the sphere. In this direction, we need the following standard result:

Lemma 2 Let $\Omega$ be a proper subdomain of the sphere. Suppose that $u$ is a nontrivial solution of the problem

$$
\left\{\begin{aligned}
\Delta u+2 u=0 & \text { in } \Omega \\
u=0 & \text { in } \partial \Omega
\end{aligned}\right.
$$

Then, $u$ does not vanish at the interior of $\Omega$.

It is also well known that for a proper subdomain $\Omega$ of the sphere, two nonpositive eigenvalues of the operator $\Delta+2$ do not exist. This means that for each surface $M$ in $\mathcal{M}$, the stability index plus the nullity is at most one. A direct consequence of the last Lemma is the following

Theorem 1 Any surface $M$ in $\mathcal{M}$ is nondegenerate. In particular, the space $\mathcal{M}$ is a one-dimensional real analytic manifold whose tangent space at $M$ is $\mathcal{J}(M)$.

Proof. The flat surface $M_{0} \in \mathcal{M}$ is clearly nondegenerate. In the nonflat case, the nondegeneracy condition for this problem holds if we know that the set of functions in the bounded nullspace of the operator $L=\Delta+2$ for a proper domain of the sphere, that vanish at the boundary of $\Omega$ and at an interior point reduces to zero. But this is true by Lemma 2. Now the smoothness of $\mathcal{M}$ follows from the results in [15], Theorem 4. Note that as we are considering only surfaces contained in the upper halfspace, the differentiable structure of $\mathcal{M}$ around $M_{0}$ identifies with $[0, \varepsilon[$ for $\varepsilon>0$.

Remark 1 When $\Gamma$ is a round circle, it is well-known that the space $\mathcal{M}$ is nothing but a collection of proper connected pieces of catenoids. This fact can be also obtained by noticing that the function $\operatorname{det}\left(p, N, e_{3}\right), p$ being the position vector on the surface and $N$ its Gauss map, is a bounded Jacobi function that vanishes at the boundary and at the end, thus nondegeneracy forces $\operatorname{det}\left(p, N, e_{3}\right)$ to vanish everywhere.

\section{The compactness theorem.}

In this Section we show a compactness result for our family of surfaces, that will play a fundamental role in the final description of the space. Other references for compactness 
can be found, for example, in Choi and Schoen [1], Ros [17] and White [21]. We begin with following uniform area bound, whose proof follows from the co-area formula, see for instance Korevaar, Kusner and Solomon [5] for a related situation:

Lemma 3 Given $p \in \mathbb{R}^{3}$, there exists a positive number $r_{0}=r_{0}(p)$ such that for all $M \in \mathcal{M}$,

$$
\text { Area }(M \cap B(p, r)) \leq \pi r^{2}+\frac{1}{2} \int_{q \in \Gamma}\langle q-p, \eta\rangle d s, \quad \forall r>r_{0}(p),
$$

where $\eta$ denotes the exterior conormal vector along the boundary.

Now we are ready to prove the main result of this Section.

Theorem 2 For any sequence $\left\{M_{n}\right\}_{n}$ in $\mathcal{M}$, one of the following possibilities holds:

i) There exists a subsequence of $\left\{M_{n}\right\}_{n}$ that converges uniformly on compact sets of $\mathbb{R}^{3}$ (with multiplicity one) to a surface in $\mathcal{M}$, or

ii) There exists a unique singular point $p$ in the bounded component of $\left\{x_{3}=0\right\}-\Gamma$ such that $\left\{M_{n}\right\}_{n}$ converges uniformly on compact subsets of $\mathbb{R}^{3}-\{p\}$ to the plane $\left\{x_{3}=0\right\}$, with multiplicity one in the unbounded component of $\left\{x_{3}=0\right\}-\Gamma$ and multiplicity two in the bounded component of $\left\{x_{3}=0\right\}-[\Gamma \cup\{p\}]$.

Proof. The argument divides in two cases, depending on the behavior of the Gauss curvature of the surfaces $M_{n}$.

FIRST CASE: The Gaussian curvatures $K_{n}$ of $M_{n}$ are uniformly bounded.

Recall that the boundary of $M_{n}$ is fixed and the surfaces $M_{n}$ lie in the upper halfspace. Moreover, we have local area estimates by Lemma 3. General compactness arguments, see Theorem 4.2.1 in [16] for the case without boundary and White [21] for nonempty boundary, guarantee that there exists a (possibly nonconnected) complete embedded minimal surface $M_{\infty} \subset\left\{x_{3} \geq 0\right\}$ such that for each component $\Sigma$ of $M_{\infty}$ it holds that either $\partial \Sigma=\Gamma$ or $\partial \Sigma=\varnothing$, at least one of the components of $M_{\infty}$ has nonvoid boundary, and after passing to a subsequence, the surfaces $M_{n}-\Gamma$ converge uniformly on compact sets of $\mathbb{R}^{3}-\Gamma$ to $M_{\infty}$ with finite multiplicity (which depends on the connected component of $M_{\infty}$ ). In particular, any component of $M_{\infty}$ must have genus zero and absolute total curvature at most $4 \pi$ (the control on the genus follows because the components of $M_{\infty}$ are finitely covered by surfaces of genus zero).

Let $\bar{\Sigma}=\Sigma \cup \Gamma$ be the closure of a component of $M_{\infty}$ with $\partial \Sigma=\Gamma$. Assume firstly that $\Sigma$ is not flat. By the maximum principle, $\bar{\Sigma}$ meets $\left\{x_{3}=0\right\}$ only along $\Gamma$, thus it is a connected complete embedded minimal surface with genus zero and finite total curvature contained in the upper halfspace, with boundary $\partial \bar{\Sigma}=\Gamma$. In this setting, Theorem 2 in [13] 
implies that $\bar{\Sigma}$ is an annulus and therefore, $\bar{\Sigma} \in \mathcal{M}$. Fix $c>0$. By Lemma 1 , the level curve $\bar{\Sigma} \cap\left\{x_{3}=c\right\}$ is a strictly convex curve and the same holds with $M_{n} \cap\left\{x_{3}=c\right\}$, hence the multiplicity of the limit $\left\{M_{n}\right\}_{n} \rightarrow \Sigma$ is one. Moreover, the annulus $M_{n} \cap\left\{0 \leq x_{3} \leq c\right\}$ can be chosen arbitrarily close to $\bar{\Sigma} \cap\left\{0 \leq x_{3} \leq c\right\}$ for any $\varepsilon>0$ small. So, $M_{\infty}$ has no other components with nonempty boundary. If $M_{\infty}$ had a component $\Sigma^{\prime}$ with $\partial \Sigma^{\prime}=\varnothing$, then $\Sigma^{\prime}$ would be a horizontal plane in $\left\{x_{3}>0\right\}$ by the Halfspace Theorem [4]. In particular, $\Sigma^{\prime}$ would intersect $\Sigma$, a contradiction. As consequence, we have arrived to item $i$ ) in the statement of the Theorem.

Now assume that $M_{\infty}$ is flat. Then, the normal vector of $M_{n}$ along $\Gamma$ necessarily takes values arbitrarily close to the vertical directions. Suppose that after passing to a subsequence, the horizontal projection of $M_{n}$ locally around the boundary lies at the interior of $\Gamma$ for all $n$ large. Then, the open flat disk $D$ enclosed by $\Gamma$ is a component of $M_{\infty}$. Moreover, a part of $M_{n}$, containing $\Gamma$, must be a graph over $\bar{D}=D \cup \Gamma$, which would imply the $M_{n}$ is compact. As consequence of this contradiction, we can assume that for all $n$ large the horizontal projection of $M_{n}$ locally around the boundary lies outside $\bar{D}$. In this setting, Lemma 1 gives that all the $M_{n}$ are global graphs over $M_{0}$. Therefore, $\Sigma$ must be $M_{0}, M_{\infty}$ has no other components and the multiplicity of the limit $\left\{M_{n}\right\}_{n} \rightarrow M_{0}$ is one. In summary, only item $i$ ) of our Theorem can hold in this first case.

SECOND CASE: The Gaussian curvatures $K_{n}$ are not uniformly bounded.

Take a sequence $p_{n} \in M_{n}$ where $\left|K_{n}\right|$ reaches its maximum. By hypothesis, $\left|K\left(p_{n}\right)\right| \rightarrow \infty$. Expanding homothetically each $M_{n}$ we get a new minimal surface $M_{n}^{\prime}$ passing through $0 \in \mathbb{R}^{3}$, whose absolute Gaussian curvature attains its maximum at the origin with value one. Moreover, the boundary $\partial M_{n}^{\prime}$ is a convex curve contained in a horizontal plane at nonpositive height depending on $n$, and the spherical image of each $M_{n}^{\prime}$ coincides with the one of $M_{n}$, which has area less than $4 \pi$. Note also that as the length of $\partial M_{n}^{\prime}$ becomes arbitrarily large, Lemma 3 no longer insures uniform area estimates for $M_{n}^{\prime}$. Nevertheless, $\left\{M_{n}^{\prime}\right\}_{n}$ is a sequence of properly embedded minimal surfaces with boundary, with uniform estimates of the Gaussian curvature and an accumulation point at the origin. Under these conditions, Theorem 4.2.2 in [16] (with suitable modifications to deal with the boundaries, see [21]) implies that, after passing to a subsequence, there exists a connected embedded minimal surface $M_{\infty}^{\prime} \subset \mathbb{R}^{3}$ (possibly with boundary), which is contained in the accumulation set of $\left\{M_{n}^{\prime}\right\}_{n}$, it passes through the origin with absolute Gaussian curvature one at that point, and every divergent path in $M_{\infty}^{\prime}$ either diverges to the boundary of $M_{\infty}^{\prime}$ or has infinite length. Moreover, $M_{\infty}^{\prime}$ is produced by analytic continuation, starting at the accumulation point $0 \in \mathbb{R}^{3}$, of limits of graph pieces of the surfaces $M_{n}^{\prime}$. Therefore, $M_{\infty}^{\prime}$ has total curvature not greater than $4 \pi$.

Now we discuss two possibilities: assume firstly that $\partial M_{\infty}^{\prime}=\varnothing$. In this situation, $M_{\infty}^{\prime}$ is a connected complete embedded nonflat minimal surface in $\mathbb{R}^{3}$ with absolute total curvature at most $4 \pi$, hence a catenoid. As the Gauss map of each $M_{n}^{\prime}$ omits the vertical 
directions, $M_{\infty}^{\prime}$ must be a vertical catenoid. Coming back to the original scale, we have proved that there exists a sequence of balls $B_{n}$ which radii converge to zero in such a way that by suitable rescaling, the portion of $M_{n}$ inside this balls converges to a vertical catenoid when $n$ goes to infinity. As the absolute Gaussian curvature of $M_{n}$ is at most $4 \pi$, this blowing up process can occur at most once, but a priori the sequence of centers of the balls $B_{n}$ could diverge. Let us prove that this is not the case: more precisely, we will check that the limit of $B_{n}$ is a point $p$ lying in the flat disk $D$ bounded by $\Gamma$. Working with $n$ large enough, we can assume that $\overline{B_{n}} \cap \Gamma=\varnothing$ for all $n$. As the portion of $M_{n}$ inside $B_{n}$ is arbitrarily close to a shrunk image of the intersection of a big ball with a vertical catenoid, it follows that $\partial\left(M_{n} \cap B_{n}\right)$ consists of two curves $\alpha_{n}, \widetilde{\alpha}_{n}$ close to round horizontal circles in $\partial B_{n}, \alpha_{n}$ lying below $\widetilde{\alpha}_{n}$. Furthermore, the Gauss map of $M_{n} \cap B_{n}$ covers the whole sphere minus two small neighborhoods of the vertical directions. In particular, the projection $\pi$ of $M_{n} \cap\left(\mathbb{R}^{3}-B_{n}\right)$ on the plane $\left\{x_{3}=0\right\}$ is a local diffeomorphism. As $M_{n}$ intersects every horizontal plane in a single curve, we deduce that the portion of $M_{n}$ below $\alpha_{n}$ is a compact annulus $A_{n}$ with boundary $\Gamma \cup \alpha_{n}$. Analogously, the piece of $M_{n}$ above $\widetilde{\alpha}_{n}$ is a noncompact annulus $\widetilde{A}_{n}$ with boundary $\widetilde{\alpha}_{n}$. Gluing smoothly $A_{n}$ with an almost horizontal disk $\Delta_{n}$ along $\alpha_{n}$, we extend $\pi$ to a local diffeomorphism from $\overline{A_{n}} \cup \Delta_{n}$ to a compact disk in $\left\{x_{3}=0\right\}$, hence a global diffeomorphism. In particular, $A_{n}$ is a graph over its projection in $\left\{x_{3}=0\right\}$ and $\pi\left(\alpha_{n}\right)$ lies in the interior of the disk $D$. In a similar way we deduce that $\widetilde{A}_{n}$ is a graph over the noncompact component of $\left\{x_{3}=0\right\}-\pi\left(\widetilde{\alpha}_{n}\right)$. Moreover, the Gauss map of $A_{n}$ is an open map which image reduces to an arbitrarily small disk around the North or South pole in the sphere and $\Gamma \subset \partial A_{n}$ is at height zero, thus we conclude that $\alpha_{n}$ converges to a point $p \in D$, and the same holds with the limit of the balls $B_{n}$. Finally, the convergence of $\left\{M_{n}\right\}_{n}$ to $\left\{x_{3}=0\right\}$ on compact subsets of $\mathbb{R}^{3}-\{p\}$ with the multiplicities stated in our Theorem follows directly from the above description of $M_{n}$ as $A_{n} \cup \widetilde{A}_{n} \cup\left(M_{n} \cap B\right)$.

To finish the proof, we check that $\partial M_{\infty}^{\prime} \neq \varnothing$ is impossible. If $\partial M_{\infty}^{\prime}$ is nonvoid, then it must be the limit of arbitrarily large homothetic images of $\Gamma$, hence it is a straight line $L$ in a horizontal plane at height $c \leq 0$. As $M_{\infty}^{\prime}$ is a nonflat minimal surface contained in $\left\{x_{3} \geq c\right\}$, the maximum principle implies that $M_{\infty}^{\prime}$ meets $\left\{x_{3}=c\right\}$ only along $L$. Thus, the reflection of $M_{\infty}^{\prime}$ through $L$ will produce a connected embedded complete minimal surface $S$ without boundary (embeddedness of $S$ follows because the reflected copy of $M_{\infty}^{\prime}$ lies entirely below the height $c$ ). Moreover, $S$ contains a straight line and has absolute total Gaussian curvature less than or equal to $8 \pi$. As this surface cannot exist (see for instance [3]), we get the desired contradiction. 


\section{The final description of the space of surfaces.}

We will start this last Section by dealing with the connectedness of the one dimensional manifold $\mathcal{M}$. Thanks to the works in $[14,15]$, we dispose of an analytic map log: $\mathcal{M} \rightarrow \mathbb{R}$ that assigns to each surface $M \in \mathcal{M}$ the logarithmic growth of its end. Moreover, the differential of $\log$ at $M$ is given by $u \in \mathcal{J}(M) \mapsto D \log _{M}(u)=\log (u)$, where $\log (u)$ stands for the logarithmic growth of the Jacobi function $u \in \mathcal{J}(M)$, that is

$$
u(w)=-\log (u)\left\langle N, e_{3}\right\rangle \log |w|+h(w), \quad 0<|w|<\varepsilon,
$$

where $w$ is the graph coordinate around the end, $N$ is the Gauss map of $M$ and $h$ is a smooth function in $\{|w|<\varepsilon\}$ (see details in [14]). Hence we obtain directly the equivalence of the following facts:

a. $M$ is a critical point of $\log : \mathcal{M} \rightarrow \mathbb{R}$.

b. $\mathcal{J}(M)=\mathcal{K}(M)$.

c. 0 is an eigenvalue of $L=\Delta+2$ on the compactified surface.

d. 0 is the first eigenvalue of $L=\Delta+2$ on the compactified surface.

As usual, a surface $M \in \mathcal{M}$ is said to be strictly stable if the first eigenvalue of $L=\Delta+2$ on the compactified surface is positive.

Proposition 1 Any stable surface $M \in \mathcal{M}$ can be joined to the flat surface $M_{0}$ by a curve in $\mathcal{M}$ which is entirely formed by strictly stable surfaces (up to the initial $M$ if this initial surface has first Jacobi eigenvalue zero), where the logarithmic growth is strictly decreasing.

Proof. Suppose firstly that $M$ is strictly stable. By the equivalence above, the curve $\mathcal{M}$ can be locally parametrized by the logarithmic growth, hence we will write it as $\left\{M_{s}\right\}_{s}$ where $s$ denotes the logarithmic growth of the surface $M_{s}$ near $M=M_{s_{0}}$. Consider the direction in which log decreases and denote by $g_{s}$ the $\overline{\mathbb{C}}$-valued Gauss map of $M_{s}$. We claim that as $\log$ decreases, the spherical image $g_{s}\left(M_{s}\right)$ becomes smaller, that is, $g_{s_{1}}\left(M_{s_{1}}\right) \subset g_{s_{2}}\left(M_{s_{2}}\right)$ if $s_{1}>s_{2}$ :

Let $\psi_{s}: M \rightarrow \mathbb{R}^{3}$ be the variation of $M_{s_{0}}$ that gives $M_{s}$ (so $\psi_{s_{0}}$ is the identity), and denote by a dot the derivative at $s=s_{0}$. Locally around the boundary $\Gamma, \psi_{s}$ can be supposed to be a normal variation, $\psi_{s}(p)=p+v_{s}(p) N(p), N$ being the Gauss map of $M$, thus $\dot{v}=u$ is a generator of $\mathcal{J}(M)$ with $\log (u)=D \log _{M}(u)<0$. It follows that $\dot{N}=-\nabla u$ and as $u$ vanishes at $\Gamma$,

$$
\left.\dot{N}\right|_{\Gamma}=-\frac{\partial u}{\partial \eta} \eta
$$


$\eta$ being the exterior unit conormal vector to $\Gamma$. Let us see that the partial derivative above is strictly positive on $\Gamma$. Note that the stability of $M$ implies that $u$ has constant sign on $M$ (in fact, this also holds if the first Jacobi eigenvalue of $M$ is zero). As $N$ takes the value $e_{3}=(0,0,1)$ at the end and $\log (u)<0$, equation (1) implies that $u$ is negative near $w=0$, thus it is negative everywhere on $M$. This means that $u$ reaches $\Gamma$ with strictly positive derivative respect to the exterior conormal vector, as desired. As $\eta$ points to the lower radial direction from the North Pole of the sphere $\mathbb{S}^{2}$, we have that $\dot{N}$ has the upper radial direction along the boundary $\Gamma$ and our claim is proved.

Hence, when moving toward the decreasing direction of the logarithmic growth we find strictly stable surfaces near $M$. Repeating the argument above, we find by prolongation a maximal curve starting from $M$ which is entirely formed by strictly stable surfaces. By the compactness Theorem 2, the end point of this curve is $M_{0}$ (the "singular point" case is impossible in our situation because of the stability of the elements in the curve). This finishes the proof in the strictly stable case.

If $M$ has first Jacobi eigenvalue zero, we have no longer a local parametrization of $\mathcal{M}$ by the logarithmic growth, but we know from the equivalence before this Proposition that the generator $u$ of the tangent space $\mathcal{J}(M)$ is bounded and by Lemma 2 , it has no zeros on $M-\Gamma$. So we can take this generator with negative sign everywhere and consider the deformation $\left\{M_{s}: s \in\right] s_{0}-\varepsilon, s_{0}+\varepsilon[\} \subset \mathcal{M}$ of the original surface $M=M_{s_{0}}$ having $u$ as normal part of its variational field. Then the argument above remains valid and we deduce that for $s \in] s_{0}, s_{0}+\varepsilon[$ we find only strictly stable surfaces. Now the proof finishes by applying the former case.

In [15], the authors exhibited an analytic immersion of the one-dimensional manifold $\mathcal{M}-\left\{M_{0}\right\}$ into $\mathbb{R}^{2}$ by means of the map $f_{c}(M)=\left(\log (M)\right.$, height $\left.{ }_{c}(M)\right)$, where height $_{c}(M)$ denotes the height of the center of the half-catenoid asymptotic to $M$. Moreover they proved that, when restricted to the subset of strictly stable surfaces, the image of $f_{c}$ is a locally strictly convex curve. Clearly $f_{c}$ can be continuously extended to $M_{0} \in \mathcal{M}$ by defining $f_{c}\left(M_{0}\right)=(0,0)$. We now give a more precise description of the shape of this planar curve.

Theorem 3 The one-dimensional manifold $\mathcal{M}$ is connected and can be analytically embedded as a curve in $\mathbb{R}^{2}$ by $M \in \mathcal{M} \mapsto f_{c}(M)=\left(\log (M)\right.$, height $\left.{ }_{c}(M)\right)$. This curve starts at the origin $(0,0)$ (that corresponds to the exterior of $\Gamma$ in $\left\{x_{3}=0\right\}$ ) with strictly increasing logarithmic growth, parametrizing the strictly stable surfaces of $\mathcal{M}$ as a strictly convex curve until it reaches its maximum first coordinate at a unique critical surface $M_{a_{0}} \in \mathcal{M}$ with first Jacobi eigenvalue zero. Next, the curve has strictly decreasing logarithmic growth and parametrizes the set of unstable surfaces with boundary $\Gamma$, tending to the origin as a "double covering" of the plane $\left\{x_{3}=0\right\}$. 
Proof. Call $C_{M_{0}}$ to the connected component of $M_{0}$ in $\mathcal{M}$. We know by Proposition 1 that the subset $\mathcal{S} \subset \mathcal{M}$ of stable surfaces is contained in $C_{M_{0}}$. By the compactness Theorem 2, the map $\log : \mathcal{M} \rightarrow \mathbb{R}$ is bounded and there exists a surface $M_{a_{0}} \in \mathcal{M}$ where log attains its maximum in $C_{M_{0}}$, so $M_{a_{0}}$ has first Jacobi eigenvalue zero. On the other hand, any surface in $\mathcal{M}$ with first Jacobi eigenvalue zero can be joined by strictly stable surfaces to $M_{0}$ by Proposition 1 , hence $M_{a_{0}}$ is the only surface of this type in $\mathcal{M}$. If $M$ is any surface in $\mathcal{M}$ and $C_{M}$ denotes its connected component, the compactness Theorem 2 insures the existence of a surface where log achieves its maximum among the surfaces in $C_{M}$. This maximum has first Jacobi eigenvalue zero so it has to be $M_{a_{0}}$. This gives the connectedness of $\mathcal{M}$.

To finish the proof, we will demonstrate that $f_{c}\left(\mathcal{M}-\left\{M_{0}\right\}\right)$ is embedded. As the piece of strictly stable surfaces is convex when seeing it as a curve in $\mathbb{R}^{2}$ and the log map is strictly decreasing on the set of unstable surfaces (as one-dimensional manifold, $\mathcal{M}$ inherits a natural order starting at $M_{0}$ ), it only remains to see that a strictly stable surface cannot have the same logarithmic growth and height that an unstable one. Suppose $M, M^{\prime} \in \mathcal{M}-\left\{M_{0}\right\}, M$ being strictly stable and $M^{\prime}$ unstable, such that $\log (M)=\log \left(M^{\prime}\right)$ and height $\mathbf{h}_{c}(M)=$ height $_{c}\left(M^{\prime}\right)$. Therefore the surfaces are asymptotic and the maximum principle at infinity $[7,8]$ insures that $M^{\prime}$ does not lie above $M$ outside any compact set (and vice versa). As $M$ is strictly stable and the three-dimensional manifold whose boundary is $M \cup M_{0}$ is foliated by strictly stable surfaces in $\mathcal{M}$, we deduce that there exists a first contact point between $M^{\prime}$ and a stable surface $M^{\prime \prime} \in \mathcal{M}$ between $M$ and $M_{0}$, a contradiction. This finishes the proof of the Theorem.

Remark 2 When $\Gamma$ is a circle of radius one, the planar curve $f_{c}(\mathcal{M})$ is symmetric respect to the log-axis, the stable surfaces in $f_{c}(\mathcal{M})$ are parametrized by $t \in[0,1] \mapsto$ $\left(t, t \log \frac{1-\sqrt{1-t^{2}}}{t}\right)$, and the unique critical annulus with first Jacobi eigenvalue is the halfcatenoid having its waist circle as boundary and logarithmic growth one, which corresponds to the point where $f_{c}(\mathcal{M})$ meets the log-axis, see Figure 1.

An interesting open problem is that of decide whether the curve $f_{c}(\mathcal{M})$ is globally convex. As a direct consequence of Theorem 3, we deduce a statement about the "exterior Plateau problem" in the line of the works by Meeks and White $[9,10]$ : Consider a convex Jordan curve $\Gamma \subset\left\{x_{3}=0\right\}$ and a real positive number $a$. We are interested in finding all complete embedded minimal surfaces $\Sigma \subset\left\{x_{3} \geq 0\right\}$ with boundary $\Gamma$ and exactly one end with finite total curvature and logarithmic growth $a$. In this setting, it holds

Corollary 1 Let $\Gamma \subset\left\{x_{3}=0\right\}$ be a convex Jordan curve. Then, there exists a unique critical value $a_{0}$ such that one of the following possibilities holds:

1. $a>a_{0}$ and there are no properly immersed minimal surfaces in the upper halfspace with boundary $\Gamma$ and one embedded end of finite total curvature with logarithmic growth a (with or without branch points). 


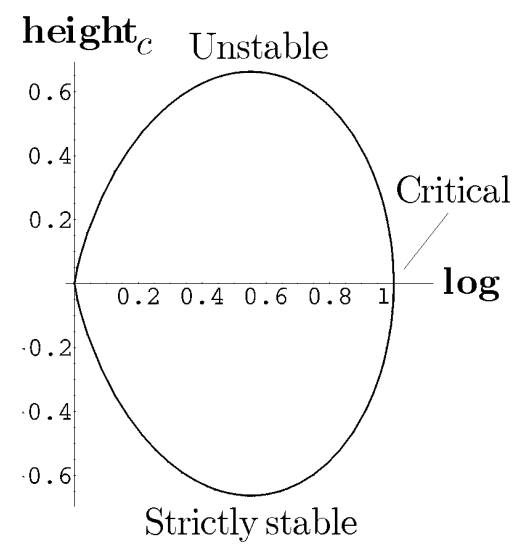

Figure 1: The curve $f_{c}(\mathcal{M})$ when $\Gamma$ is the unit circle.

2. $a=a_{0}$ and there exists exactly one minimal annulus with this boundary and logarithmic growth. In this case, this annulus has first Jacobi eigenvalue zero and there are no other (possibly branched) solutions of this Plateau problem with higher topology.

3. $0<a<a_{0}$ and there exist exactly two annuli with this boundary and this logarithmic growth, one strictly stable and one unstable (perhaps there are other solutions of this exterior Plateau problem with higher topology).

Proof. Take a minimal surface $\Sigma$ properly immersed in the upper halfspace, possibly branched, with boundary $\Gamma$ and exactly one embedded end of finite total curvature and logarithmic growth $a$. Consider the one-parameter family $\mathcal{M}=\left\{M_{t}\right\}_{t \geq 0}$ of minimal annuli with boundary $\Gamma$, so that $M_{0}$ is the exterior of this curve in the plane $\left\{x_{3}=0\right\}$, and suppose that the logarithmic growth $a$ of the end of $\Sigma$ satisfies $a \geq a_{0}$, this last one being the logarithmic growth of the unique surface in $\mathcal{M}$ with first Jacobi eigenvalue zero. Suppose also that $\Sigma \notin \mathcal{M}$. By the classical maximum principle, $\Sigma$ only cuts to $M_{t}$ along $\Gamma$ for $t>0$ small enough. We claim that for any $t$, it holds $\left(\Sigma \cap M_{t}\right)=\Gamma$. For, suppose that $A=\left\{t \geq 0:\left(\Sigma \cap M_{t}\right)-\Gamma \neq \varnothing\right\}$ is nonvoid, and consider $t_{0}=\operatorname{infimum}(A)$. Our assumption on the logarithmic growth of $\Sigma$ implies that $M_{t_{0}}-\Gamma$ and $\Sigma-\Gamma$ have a first contact point that has to occur at infinity by the classical maximum principle for minimal surfaces, so $\Sigma$ and $M_{t_{0}}$ are asymptotic, $\Sigma$ lying above $M_{t_{0}}$. This contradicts the maximum principle at infinity $[7,8]$, and our claim is proved.

To find the desired contradiction on the existence of $\Sigma$, it only remains to use that when log goes to zero by unstable surfaces $M_{t}$ strangles at the singular point, together with the property that $\Sigma \cap M_{t}=\Gamma$ for all $t$. Now the proof is complete. 
Remark 3 The last result also gives a short proof of a well-known fact: If the boundary $\Gamma$ has a symmetry, then any minimal annulus with boundary $\Gamma$ inherits this symmetry: note that the symmetry preserves both the boundary and the stability character of the annulus, and Corollary 1 implies that the number of strictly stable (resp. unstable, critical) minimal annuli with boundary $\Gamma$ is at most one). This statement was firstly proven by using Alexandrov reflection technique, see Schoen [18].

\section{References}

[1] H. Choi \& R. Schoen, The space of minimal embeddings of a surface into a three dimensional manifold of positive Ricci curvature, Invent. Math. 81 (1985) 387-394.

[2] P. Collin, Topologie et courbure des surfaces minimales proprement plongees de $\mathbb{R}^{3}$, Ann. of Math. 2nd Series 145-1 (1997) 1-31.

[3] C. Costa, Uniqueness of minimal surfaces embedded in $\mathbb{R}^{3}$ with total curvature $12 \pi$, J. Differential Geometry 30(3) (1989) 597-618.

[4] D. Hoffman \& W. H. Meeks III, The strong halfspace theorem for minimal surfaces, Invent. Math. 101 (1990) 373-377.

[5] N. Korevaar, R. Kusner \& B. Solomon, The structure of complete embedded surfaces with constant mean curvature, J. Diff. Geom. 30 (1989) 465-503.

[6] R. Krust, Remarks on the exterior Plateau problem, Duke Math. J. 59 (1) (1989) $161-173$.

[7] R. Langevin \& H. Rosenberg, A maximum principle at infinity for minimal surfaces and applications, Duke Math. J. 57 (1988) 819-828.

[8] W. H. Meeks III \& H. Rosenberg, The maximum principle at infinity for minimal surfaces in flat three manifolds, Comm. Math. Helv. 65, 2 (1990) 255-270.

[9] W. H. Meeks III \& B. White, Minimal surfaces bounded by convex curves in parallel planes, Comment. Math. Helv. 66(2) (1991) 263-278.

[10] W. H. Meeks, III \& B. White, The space of minimal annuli bounded by an extremal pair of planar curves, Communications in Anal. and Geom. 1(3) (1993) 415-437.

[11] R. Osserman, A survey of minimal surfaces, vol. 1, Cambridge Univ. Press, New York (1989). 
[12] J. Pérez, On singly-periodic minimal surfaces with planar ends, Trans. of the A.M.S. 349-6 (1997) 2371-2389.

[13] J. Pérez \& A. Ros, Some uniqueness and nonexistence theorems for embedded minimal surfaces, Math. Ann. 295(3) (1993) 513-525.

[14] J. Pérez \& A. Ros, The space of properly embedded minimal surfaces with finite total curvature, Indiana Univ. Math. J. 451 (1996), 177-204.

[15] J. Pérez \& A. Ros, The space of complete minimal surfaces with finite total curvature as lagrangian submanifold, Trans. of the A. M. S. 35110 (1999) 3935-3952.

[16] J. Pérez \& A. Ros, Properly embedded minimal surfaces with finite total curvature, preprint.

[17] A. Ros, Compactness of space of properly embedded minimal surfaces with finite total curvature, Indiana Univ. Math. J. 44 (1995) 139-152.

[18] R. Schoen, Uniqueness, Symmetry and embeddedness of minimal surfaces, J. Differential Geometry 18 (1983) 701-809.

[19] M. Shiffman, On surfaces of stationary area bounded by two circles, or convex curves, in parallel planes, Ann. of Math. 63 (1956) 77-90.

[20] F. Tomi \& R. Ye, The exterior plateau problem, Math. Z. 205(2) (1990) 233-245.

[21] B. White, Curvature estimates and compactness theorems in 3-manifolds for surfaces that are stationary for parametric elliptic functionals, Invent. Math. 88 (1987) 243256.

\author{
JoAquin Perez Antonio Ros \\ jperez@ugr.es aros@ugr.es \\ Departamento de Geometria y Topologia \\ Facultad de Ciencias - Universidad de Granada \\ Fuentenueva, 18071 - Granada - Spain
}

